\title{
Complementariedad y sostenibilidad en las sociedades de la prehistoria tardía en el valle de Sama, Tacna, sur del Perú
}

\author{
Complementarity and sustainability in late prehistoric societies in \\ the valley of Sama-Tacna, southern Perú
}

${ }^{1}$ Carlos Vela Velarde

ORCID: 0000-0002-3187-1840

\section{RESUMEN}

Esta investigación se basó en el supuesto básico de la presencia inca en la costa de Tacna- Perú. Se desarrollaron relaciones de complementariedad con la sociedad local y que, gracias al registro arqueológico y las evidencias ambientales, dio como resultado la posibilidad de haberse generado un desarrollo sostenible en estas sociedades de la Prehistoria Tardía en el valle de Sama. De manera experimental, un ensayo computacional propuso y coincidió con el análisis arqueológico. Esta investigación es un ejemplo de aplicación de la ciencia arqueológica al modelo económico: la complementariedad (variable independiente); otra variable es el medio ambiente. En esta investigación se propuso que estas sociedades lograron un desarrollo sostenible.

Palabras clave: Complementariedad, presencia inca, sostenibilidad.

\section{ABSTRACT}

This research was based on the basic assumption of the Inca presence on the coast of Tacna-Perú. A complementary relationship with local society was developed, therefore, thanks to the archaeological and environmental evidence, it resulted in the possibility of having generated sustainable development in these societies of late Prehistory in the Sama Valley. Experimentally, a computational test propose and coincided with archaelogy analysis. This investigation it is an example to application of the science of archaelogy to the complementary model (independent variable). Another variable in this research is the environment. This research proposes that these societies achieved sustainable development.

Keywords: complementarity, sustainability, Inca presence

${ }^{1}$ Universidad Nacional Jorge Basadre Grohmann. Tacna-Perú. E-mail: carlosvelatacna@yahoo.es

Presentado: 23/08/18, Aprobado: 27/11/19 


\section{INTRODUCCIÓN}

Existe suficiente doctrina arqueológica y etnohistórica (Lumbreras, Cúneo Vidal, Mujica, Rice, Muñoz, Schiappacasse, Stanish ó Rostowrowski, Galdos, Barriga, etc.), es decir, desde el campo arqueológico y de la etnohistoria, las ciencias sociales han elaborado los sustentos teóricos acerca de la prehistoria tardía en el sur del Perú. Esta investigación retoma dichos aportes como factores demostrativos de la presencia inca y su relación con la sociedad local en el valle de Sama. Además de la relación con los factores medioambientales como sustento de un desarrollo sostenible de estas sociedades de la prehistoria tardía en el valle de Sama.

La presente investigación abordará, mediante la ciencia arqueológica, los materiales del sitio arqueológico "Los Hornos", que se ubica en el Centro Poblado Menor Boca del Río, distrito de Sama Las Yaras, provincia de Tacna, departamento de Tacna, (de un sector denominado "Los Hornos"), así como el análisis ambiental de la cuenca del valle de Sama. Esta investigación es un ejemplo de aplicación de la ciencia arqueológica al modelo económico: La Complementariedad (Variable independiente), en donde no existieron las contradicciones de la sociedad actual. Esta teoría fue estudiada inicialmente por la etnohistoria, en base a documentos escritos, mediante la ciencia arqueológica, específicamente en el valle de Sama. La complementariedad fue el modelo esencial en las sociedades del periodo tardío (siglos XIV, $\mathrm{XV}$ ), debido a que de esa forma era posible realizar transacciones económicas más dinámicas. El registro arqueológico demuestra que no existió una plena anexión al orden incaico, es decir, el proceso de "incanización" fue limitado. Más bien, en su expansión, la población inca se instaló en lugares estratégicos con grupos de Mitmakunas o grupos dedicados a mitar, es decir, a administrar, acopiar y redistribuir la producción.

Otra variable, en esta investigación, es el medio ambiente. Para ello, se incluye un análisis ambiental de la cuenca del Sama, con datos de flora, fauna, recursos, microclimas; así como la valoración ambiental y otros parámetros que permiten desarrollar un análisis a profundidad de los aspectos ambientales, propios del valle de Sama. Además de datos ambientales asociados a los datos arqueológicos, esta información procesada nos permite sostener, que en estas sociedades se generó un desarrollo sostenible; es decir, que, en base a la colusión del sistema de Complementariedad, como modelo socioeconómico sustentado por el Medio Ambiente, es posible tener Desarrollo Sostenible.

Debido al potencial arqueológico, se decidió afrontar el estudio; así como exploraciones en el valle de Sama, desde sus nacientes en la zona de puna hasta su desembocadura en el océano Pacífico.

La presente investigación se basa en el supuesto básico de que la presencia inca en la costa de Tacna establece relaciones de complementariedad con las poblaciones locales, así como diseña una estrategia de asentamiento para realizar un control y aprovechamiento de pisos ecológicos, además de que la complementariedad es el sustento para la sostenibilidad en este valle, tanto en su dimensión ambiental como en su dimensión social.

Propone que en el valle de Sama, durante el periodo Tardío de la prehistoria, se desarrollaron sociedades de identidad local que establecieron relaciones de complementariedad con las poblaciones incas que se expandieron a este valle; y que a su vez se instalaron con asentamientos a diferente altitud sobre el nivel del mar, estableciendo un control de pisos ecológicos y logrando un desarrollo sostenible en base a los recursos naturales, los sistemas de asentamiento y las relaciones de complementariedad entre la sociedad local y la sociedad inca.

Desde el punto de vista teórico, el problema de investigación está suficientemente informado, dada la variedad de concepciones y planteamientos que ha estudiado la prehistoria tardía, sin embargo, el aspecto teórico no resuelve nuestro planteamiento de investigación. 


\section{Ubicación del problema}

Por la naturaleza del problema debe ser ubicado en el campo de las ciencias sociales, específicamente en la ciencia arqueológica, mientras por su contenido corresponde al estudio de las sociedades de la prehistoria tardía que ocuparon el litoral costero del departamento de Tacna y el valle de Sama. Son componentes de este proceso, las sociedades denominadas con Desarrollo Local Tardío o Reinos del Periodo Intermedio Tardío, y las poblaciones incaicas que en su expansión se instalaron en el valle de Sama y en la costa de Tacna, donde desarrollaron relaciones de complementariedad. Esta investigación se ubica también en el campo del desarrollo sostenible, debido a esta problemática estudiada en las sociedades del siglo XV que involucran las relaciones sociales y las relaciones ambientales.

\section{Justificación del problema}

a) Debido al potencial arqueológico del sitio se justifica la investigación, la que presenta variedad de indicadores como cerámica, líticos, arquitectura, fauna, flora, enterramientos, caminos, residuos, etc.

b) El modelo de complementariedad y el desarrollo sostenible desarrollado por la ciencia histórica y la ecología pueden ser aplicados por la ciencia arqueológica, en tal sentido, las evidencias registradas en el valle de Sama sirven para este fin. Este es un tema inédito hasta ahora.

c) Mediante este estudio se puede entender mejor el sistema de relaciones entre la sociedad local y la sociedad inca, a través de estudios etnohistóricos y/o documentales, así como por la arqueología.

d) Esta investigación permitirá un mejor reconocimiento de los materiales arqueológicos. En Tacna, los estudios arqueológicos y ambientales son muy limitados por su profundidad.

e) Esta investigación contribuye desde una perspectiva ambiental para reconocer la problemática de los diversos pisos ecológicos, sus potencialidades y los modelos de producción en el periodo prehispánico.
Constituye la primera investigación sistemática de la presencia inca en el litoral de Tacna y en el valle de Sama establece la relación cultura-ambiente.

\section{Formulación del Problema}

¿Es posible afirmar que la sociedad inca alcanzara desarrollo sostenible significativo al establecer relaciones de complementariedad con las sociedades locales asentadas en el valle de Sama?

¿Se puede calificar de óptima la política de aprovechamiento y control de pisos desarrolladas por la sociedad local y la sociedad inca en el valle de Sama?

¿Es posible establecer a las relaciones ambientales del valle de Sama como condicionantes de sostenibilidad en la población del valle de Sama?

¿Cómo influyó la presencia inca para desarrollar relaciones de complementariedad con la sociedad local como base para el desarrollo sostenible?

¿Cómo influyó la presencia inca en el aprovechamiento y control de pisos ecológicos en el valle de Sama?

¿Qué relación se puede encontrar entre el control de pisos ecológicos, la complementariedad y el desarrollo sostenible en el valle de Sama?

\section{Hipótesis}

Las relaciones de complementariedad y las condiciones medioambientales de las sociedades del valle de Sama posibilitaron que se alcance un desarrollo sostenible de nivel significativo en el periodo tardío de la prehistoria.

\section{Sub-hipótesis}

Las sociedades del valle de Sama desarrollaron relaciones de complementariedad con la población Inca las evidencias arqueológicas sugieren un desarrollo sostenible debido al sistema de relaciones de complementariedad, en lo económico, social y étnico.

La complementariedad fue el sustento socioeconómico para el desarrollo sostenible en las sociedades asentadas en el valle de Sama en el periodo tardío de la prehistoria.

El control de pisos ecológicos, el medio ambiente, el aprovechamiento de los recursos 
naturales por las poblaciones incaicas y las poblaciones locales en el valle de Sama. Fueron la base ambiental para el desarrollo sostenible.

\section{MATERIALES Y MÉTODOS}

Mediante el software estadístico Statgraphics plus versión 5.0 se realizó un ensayo, tomando como muestra los valores obtenidos en el estudio de la cerámica de "Los Hornos". Los resultados dieron como predicciones que las interrelaciones entre la sociedad local y la sociedad inca era muy ligera, es decir no logra asimilarse en el total de la sociedad y que la "curva de optimización" de la presencia inca tendía a decaer súbitamente en comparación con la curva de la sociedad local que se visualiza con una muy significativa optimización. Este ensayo permite que las variables culturales sean desarrolladas por las interrelaciones de este software.

Mediante el presente estudio se adecúa una investigación estadística aplicada a las ciencias sociales, específicamente a la arqueología, se aplica el software estadístico Statgraphics plus versión 5.0, asimismo el Excel Microsoft (2000).

\section{RESULTADOS}

Se realizó un ensayo computacional mediante el software Statgraphics Plus 5.0, para el estudio de las relaciones culturales entre la sociedad local y la sociedad inca

Mediante el software estadístico STATGRAPHICS 5.0 PLUS se realizó un ensayo, tomando como muestra los valores obtenidos en el estudio de la cerámica de "Los Hornos", los resultados dieron como predicciones que las interrelaciones entre la sociedad local y la sociedad inca era muy ligera, es decir no logra asimilarse en el total de la sociedad y que la "curva de optimización" de la presencia inca tendía a decaer súbitamente en comparación con la curva de la sociedad local que se visualiza con una muy significativa optimización. Este ensayo permite que las variables culturales sean desarrolladas por las interrelaciones de este software.

\section{a) Definición de variables}

Las variables consideradas para el presente estudio de cuantificación de la presencia cultural inca en el valle de Sama y su interacción con las sociedades locales son:

\section{b) Procesamiento de datos}

Se utilizarán los principios básicos de la estadística descriptiva, asimismo se aplica un diseño factorial simple con dos variables poblacionales $=2(\mathrm{k})$, lo cual nos permite visualizar si existen factores en la interacción de la presencia inca y la población local, la cual se establece a través de restos arqueológicos.

Para el presente estudio se aprovecha una muestra de excavación, así como de recolección superficial.

\section{c) Método analítico}

A continuación, se muestran los gráficos del software Statgraphics 5.0 plus en base a información del sitio "Los Hornos" y en el valle de Sama.

Contours of Estimated Response Surface

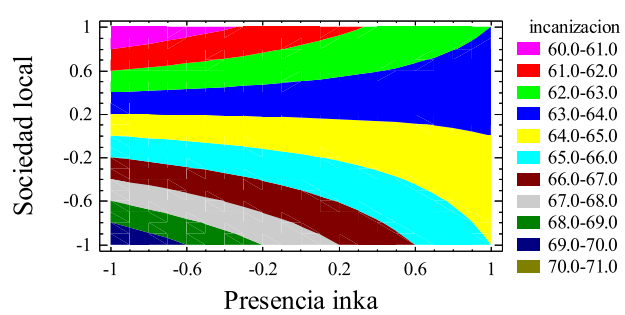

Figura 1. Gráfico que sugiere que la interacción entre sociedad local y presencia inca se dividen y no logran asimilarse.

Interpretación de Contours of Estimated Response Surface:

El factor sociedad local tiene mayor efecto significativo en las curvas de nivel en comparación a la presencia inca en la incanización, esto debido a que la disminución de la cerámica y asentamiento local, frente a la cerámica y asentamiento inca que se mantiene constante. 


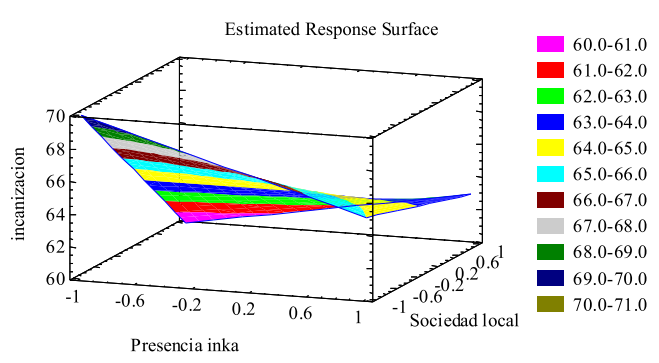

Figura 2. El factor sociedad local tiene mayor efecto significativo en la superficie respuesta

\section{Interpretación de Estimated Response Surface:}

El factor sociedad local tiene mayor efecto significativo en la superficie respuesta, tal como se visualiza en el grafico espacial que se presenta, en comparación a la presencia inca en la incanización.

Main Effects Plot for incanizacion

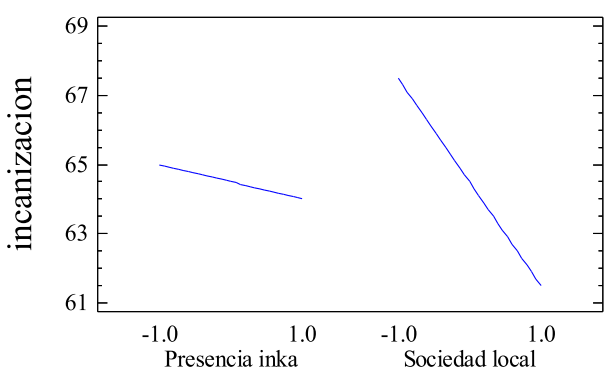

Figura 3. Efectos principales para incanización.

En la Figura 3 se observa que la presencia inca tiene una tendencia a decaer en el tiempo; mientras que la sociedad local tiene una curva que permanece y consolida su desarrollo social.

\section{Interaction Plot for incanizacion}

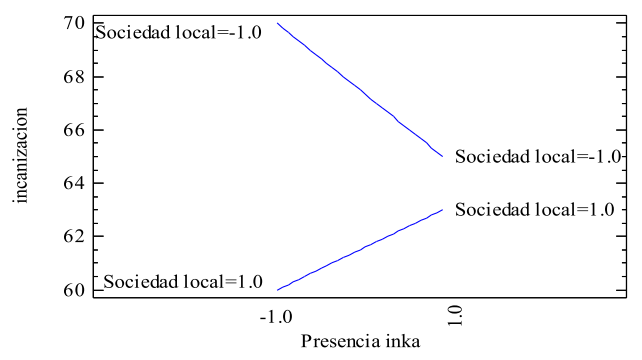

Figura 4. Trama de interacción para incanización.
La Figura 4 presenta que las interrelaciones entre la sociedad local y la presencia inca no se logran fusionar, sino más bien se alejan.

Interpretación de Main Effects Plot for Incanización:

Los factores presencia inca y sociedad local, en referencia a los niveles de estudio - cerámica inca, asentamiento inca y cerámica local, asentamiento local, respectivamente podemos establecer que disminuye la incanización verticalmente en la sociedad local que en la presencia inca. La sociedad local manifestada por la cerámica y el asentamiento local disminuye de una manera vertiginosa en comparación a la presencia inca que viene manifestada por la cerámica y el asentamiento inca de una manera casi constante. La sociedad local tiene mayor efecto significativo que la presencia inca.

\section{Pareto Chart for incanización:}

La sociedad local tiene mayor efecto significativo, para un $95 \%$ de confidencialidad, en comparación a la presencia inca. La interacción entre ambos factores tiene mayor efecto significativo la presencia local. Podemos concluir con Pareto que el que tiene mayor efecto significativo en el presente estudio es la sociedad local.

Interaction Plot for incanización:

En los rangos estudiados podemos concluir que no existe interacción entre ambos factores. Por lo que se puede manejar independientemente cada una de las variables - presencia inca y sociedad local - frente a la incanización, en los niveles establecidos para cada uno de los factores.

El presente estudio aborda el problema de la cuantificación de la presencia cultural inca en el valle de Sama y su interacción con las sociedades locales, esta investigación es enfocada desde 2 opciones de la estadística: estadística descriptiva y la estadística analítica, para esta última se aplica el software Statgraphics plus versión 5.0, además el Excel Microsoft (2000). 
La información arqueológica referida a la prehistoria tardía en el valle de Sama señala a la presencia inca como uno de sus componentes, pues este periodo desarrolló un poblamiento multiétnico entre las poblaciones locales, la presencia inca y la presencia altiplánica. En este estudio, el método analítico adecuado a la ciencia arqueológica nos permite reflexionar acerca de la presencia inca y las interacciones con la sociedad local, estableciendo coeficientes de regresión, efectos principales, interacciones, así como estimaciones de respuesta en superficie. Dichos análisis concuerdan y refuerzan estudios anteriores y plantean nuevas hipótesis de trabajo, pero en general sugieren que la presencia inca, si bien es cierto significó un limitado proceso de "incanización", desarrolló relaciones de interacción con la sociedad local.

El análisis descriptivo contribuye con cifras porcentuales que concuerdan con el desarrollo del método analítico.

La sostenibilidad se plantea por:

a) El aprovechamiento multiétnico de los espacios.

b) $\quad$ P o r l a s r e la c i o n e s d e complementariedad entre estas sociedades.

c) Por el uso racional de los diferentes pisos ecológicos y de sus recursos naturales.

d) Por el desarrollo de interrelaciones culturales de complementariedad tanto productivas como culturales.

e) Por la evidencia arqueológica que demuestra un registro muy complejo en materiales de producción de diferentes pisos ecológicos.

f) El registro arqueológico demuestra interrelaciones culturales entre la población local y la población inca.

\section{El análisis ambiental}

Tabla 1. Objetivos del Desarrollo Sostenible vs las sociedades del siglo XV-XVI en Sama

\begin{tabular}{|lc}
\hline Objetivos del Desarrollo Sostenible & Las Sociedades siglo XV-XVI Sama \\
\hline - Reactivar el crecimiento & - Aumento de la población \\
- Cambiar la calidad de crecimiento & - Relaciones de complementariedad \\
- Satisfacer las necesidades esenciales & - Aprovechamiento sostenido de los \\
- Asegurar un nivel de vida sostenible & recursos \\
para la población & - Recursos variados \\
- Conservar y compartir los recursos & - Racionalidad ecologista, \\
- Fusionar el medio ambiente y la & intercambio, reciprocidad \\
economía & - Aprovechamiento sostenido y \\
& variado, programado
\end{tabular}

Tabla 2. Condiciones para un manejo sostenible de los recursos naturales y las sociedades siglo XV-XVISama

\begin{tabular}{|c|c|}
\hline $\begin{array}{l}\text { Condiciones para un manejo } \\
\text { sostenible de los recursos naturales }\end{array}$ & Las sociedades siglo XV-XVI Sama \\
\hline $\begin{array}{l}\text { - Un sistema político que asegure una } \\
\text { participación efectiva en las } \\
\text { decisiones. }\end{array}$ & $\begin{array}{l}\text { - Sistema comunitario, rasgos de } \\
\text { parentesco. }\end{array}$ \\
\hline $\begin{array}{l}\text { - Sistema económico capaz de generar } \\
\text { excedentes. }\end{array}$ & - La Mita. \\
\hline $\begin{array}{l}\text { - Un sistema social que provea } \\
\text { soluciones a las tensiones a un } \\
\text { desarrollo no armónico y desigual. }\end{array}$ & - La complementariedad. \\
\hline $\begin{array}{l}\text { - Un sistema económico de producción } \\
\text { que respete la obligación de preservar } \\
\text { la ecología. }\end{array}$ & $\begin{array}{l}\text { - La cosmovisión ecologista, la } \\
\text { complementariedad, el intercambio. }\end{array}$ \\
\hline $\begin{array}{l}\text { - Un sistema administrativo que sea } \\
\text { flexible y tenga la capacidad de } \\
\text { corregirse }\end{array}$ & $\begin{array}{l}\text { - La Mita, los mitmaqunas, la } \\
\text { sociedad local. }\end{array}$ \\
\hline
\end{tabular}


Tabla 3. Valorización ambiental del valle de Sama, siglos XVI-XVII

\begin{tabular}{|c|c|c|c|c|}
\hline $\begin{array}{c}\text { Valor de Uso } \\
\text { Directo }\end{array}$ & $\begin{array}{c}\text { Valor de } \\
\text { Uso Indirecto }\end{array}$ & $\begin{array}{l}\text { Valor de } \\
\text { Opción }\end{array}$ & $\begin{array}{l}\text { Valor } \\
\text { Legado }\end{array}$ & $\begin{array}{c}\text { Grupos } \\
\text { Étnicos } \\
\text { vinculados }\end{array}$ \\
\hline $\begin{array}{l}\text { Pesca } \\
\text { Agricultura } \\
\text { Ganadería } \\
\text { Recolección } \\
\text { Energía } \\
\text { Transporte } \\
\text { Madera } \\
\text { Caza }\end{array}$ & $\begin{array}{l}\text { Retención de } \\
\text { nutrientes. } \\
\text { Recarga de } \\
\text { acuíferos. } \\
\text { Alimentación } \\
\text { del } \\
\text { ecosistema. } \\
\text { Estabilización } \\
\text { climática. } \\
\text { Control del } \\
\text { drenaje } \\
\text { fluvial. }\end{array}$ & $\begin{array}{l}\text { Usos futuros. } \\
\text { Potenciales. } \\
\text { Valor futuro de } \\
\text { producción. } \\
\text { Aumento de la } \\
\text { producción. } \\
\text { Mejoras en la } \\
\text { calidad } \\
\text { ambiental. } \\
\text { Aumento de } \\
\text { población. } \\
\text { Investigación. }\end{array}$ & $\begin{array}{l}\text { Biodiversidad } \\
\text { Cultura } \\
\text { Herencia } \\
\text { Paisaje }\end{array}$ & $\begin{array}{l}\text { San Miguel } \\
\text { Maytas } \\
\text { Gentilar } \\
\text { Kolla } \\
\text { Saxamar } \\
\text { Inca }\end{array}$ \\
\hline
\end{tabular}

Valor de Uso Directo: necesidades de subsistencia de las comunidades locales

Valor de Uso Indirecto: Funciones ecológicas o eco-sistémicas, cumplen rol regulador

Valor de Opción: valor potencial para uso futuro

Valor Legado: recurso para generaciones futuras

Tabla 4. Contexto Ambiental Valle de Sama

\begin{tabular}{|c|c|c|c|c|c|c|c|}
\hline $\begin{array}{c}\text { Entorno } \\
\text { Físico }\end{array}$ & $\begin{array}{c}\text { Uso de } \\
\text { Recursos }\end{array}$ & $\begin{array}{c}\text { Bio- } \\
\text { diversidad }\end{array}$ & $\begin{array}{c}\text { Población } \\
\text { Étnicos }\end{array}$ & Energía & $\begin{array}{c}\text { Patrón } \\
\text { Asentamiento }\end{array}$ & $\begin{array}{l}\text { Propiedad } \\
\text { de la Tierra }\end{array}$ & $\begin{array}{c}\text { Transporte } \\
\mathbf{y} \\
\text { movilidad }\end{array}$ \\
\hline $\begin{array}{l}\text { Fauna } \\
\text { Flora } \\
\text { Fuente de } \\
\text { agua } \\
\text { Calidad } \\
\text { de agua } \\
\text { catástrofes }\end{array}$ & $\begin{array}{l}\text { agricultura } \\
\text { uso del } \\
\text { agua } \\
\text { pastoreo } \\
\text { pesca } \\
\text { agricultura } \\
\text { Lomas }\end{array}$ & $\begin{array}{l}\text { Especies } \\
\text { endémicas } \\
\text { R. Mar, } \\
\text { Valle } \\
\text { costero, } \\
\text { valle } \\
\text { serrano, } \\
\text { Lomas }\end{array}$ & $\begin{array}{l}\text { Sociedad } \\
\text { local } \\
\text { Presencia } \\
\text { Inca }\end{array}$ & $\begin{array}{l}\text { Maderas, } \\
\text { biomas, } \\
\text { guano de } \\
\text { isla, } \\
\text { estiércol }\end{array}$ & $\begin{array}{l}\text { Control de } \\
\text { pisos } \\
\text { ecológicos, } \\
\text { transversalidad } \\
\text { Horizontal, } \\
\text { sitios de } \\
\text { pescadores, } \\
\text { agricultores, } \\
\text { ganaderos }\end{array}$ & $\begin{array}{l}\text { Sistema } \\
\text { comunitario } \\
\text { Sistema } \\
\text { kurakas } \\
\text { smitmakunas }\end{array}$ & $\begin{array}{l}\text { Caminos de } \\
\text { arrieros }\end{array}$ \\
\hline
\end{tabular}

Tabla 5.Dimensión ecológica

\begin{tabular}{|c|c|}
\hline $\begin{array}{l}\text { DIMENSIÓN } \\
\text { ECOLÓGICA }\end{array}$ & $\begin{array}{l}\text { CUENCA del río } \\
\text { SAMA }\end{array}$ \\
\hline $\begin{aligned} & \text { Estabilidad } \\
& \text { - } \text { Resistencia } \\
& \text { - } \text { Resilencia }\end{aligned}$ & $\begin{array}{ll}\text { - } & \text { Resistencia a } \\
\text { sequías, } \\
\text { inundaciones } \\
\text { - } \\
\text { Recuperación de } \\
\text { habitats, nichos } \\
\text { - } \\
\text { Recursos } \\
\text { hídricos } \\
\text { - } \\
\text { Flora, Fauna, } \\
\text { Recursos } \\
\text { - } \begin{array}{l}\text { Diversidad de } \\
\text { procesos } \\
\text { ecológicos }\end{array}\end{array}$ \\
\hline
\end{tabular}

Tabla 6. Dimensión económica

\begin{tabular}{|c|c|}
\hline $\begin{array}{l}\text { DIMENSIÓN } \\
\text { ECONÓMICA }\end{array}$ & CUENCA del río SAMA \\
\hline Productividad & $\begin{array}{l}\text { Agricultura, pastoreo, pesca, } \\
\text { extracción. }\end{array}$ \\
\hline Eficiencia & $\begin{array}{l}\text { Mita, sistema comunal, } \\
\text { excedentes }\end{array}$ \\
\hline Opcionalidad & Racionalidad, ecologista. \\
\hline
\end{tabular}

Tabla 7. Dimensión social

\begin{tabular}{ll}
$\begin{array}{l}\text { DIMENSIÓN } \\
\text { SOCIAL }\end{array}$ & CUENCA del río SAMA \\
\hline Igualdad & $\begin{array}{l}\text { Complementariedad, } \\
\text { intercambio. }\end{array}$ \\
Autonomía & $\begin{array}{l}\text { Intercambio, reciprocidad, } \\
\text { complementariedad. }\end{array}$
\end{tabular}


Tabla 8. Sitios arqueológicos en el valle de Sama, su filiación yubicación en el valle

$\begin{array}{lll}\text { Sitio } & \text { Filiación } & \text { Piso ecológico } \\ \text { Hornos } & \text { inca } & \text { litoral } \\ \text { Tomollo } & \text { local } & \text { litoral } \\ \text { Quebrada B. } & \text { inca/local } & \text { litoral } \\ \text { Balconcillo } & \text { inca/local } & \text { litoral } \\ \text { Calvario } & \text { local } & \text { litoral } \\ \text { Siquina } & \text { local } & \text { valle bajo/yunga } \\ \text { Vituña } & \text { local } & \text { valle bajo/yunga } \\ \text { Amapaya } & \text { Local } & \text { valle bajo/yunga } \\ \text { Yalata } & \text { inca } & \text { valle bajo/yunga } \\ \text { Pampa Julia } & \text { inca } & \text { valle bajo/yunga } \\ \text { Buenavista } & \text { Local } & \text { valle bajo/yunga } \\ \text { Sama Antigua } & \text { inca } & \text { valle medio/yunga } \\ \text { Tala } & \text { inca } & \text { valle medio/yunga } \\ \text { Paramarca } & \text { inca } & \text { valle alto/quechua } \\ \text { Sitajara } & \text { inca/local } & \text { valle alto/quechua } \\ \text { Susapaya } & \text { inca/local } & \text { valle alto/quechua } \\ \text { Yabroco } & \text { local } & \text { valle alto/quechua } \\ \text { Pucara } & \text { inca/local } & \text { valle alto/quechua } \\ \text { Capanique } & \text { local } & \text { valle alto/quechua }\end{array}$

Tabla 9. Análisis de materiales arqueológicos de Los Hornos y relación ecológica

\section{Material Procedencia Origen P. ecológico}

$\begin{array}{llll}\text { Cerámica } & \text { Valle } & \text { Mineral Yunga/quechua } \\ \text { Madera } & \text { valle } & \text { vegetal yunga/quechua } \\ \text { Conchas } & \text { mar } & \text { animal marino }\end{array}$

Esp.

Pescado mar animal marino

h.lobo mar mar animal marino
Algas mar vegetal marino
h. ave mar animal marino

\section{valle}

alto/pu

Camélido na animal quechua/puna

s. maíz valle vegetal yunga/quechua

s.zapallo valle vegetal yunga

s.calabaza Valle vegetal yunga

Ají Valle vegetal yunga

plumas ave mar animal marino

Chañar valle vegetal yunga

vegetal/

Textiles valle animal yunga/quechua

Cucharas valle vegetal yunga/quechua

esteras valle vegetal yunga/quechua

frag. Cobre valle mineral quechua/puna

puntas

flecha valle mineral yunga

Batanes valle mineral yunga

"quincha" valle vegetal yunga

Carrizo valle vegetal yunga

valle/li

Barro toral mineral yunga

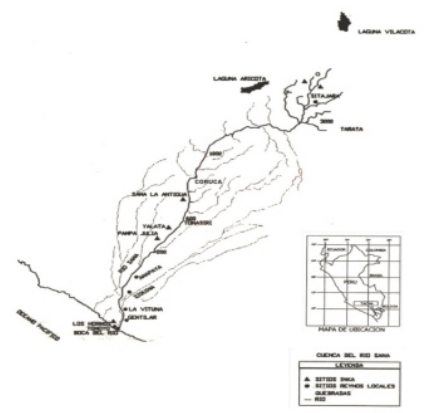

Figura 5. Valle de Sama y ubicación de sitios arqueológicos tardíos, cuenca del valle de Sama, sitios inca y sitios locales, a lo largo del valle de Sama se ubican sitios de filiación inca, así como de las sociedad locales. 


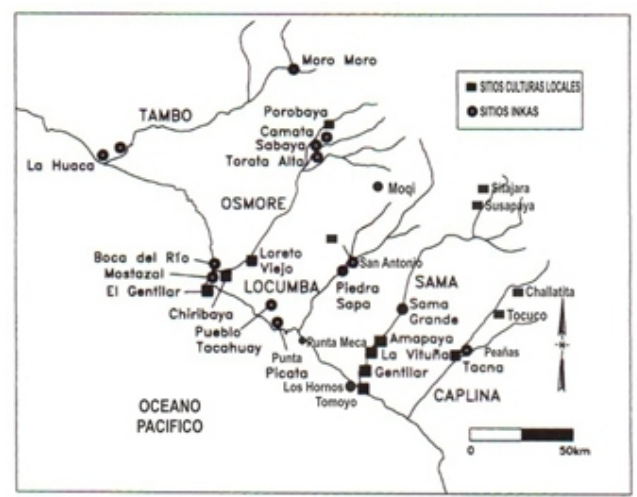

Figura 6. Sitios arqueológicos en valles del sur del Perú.

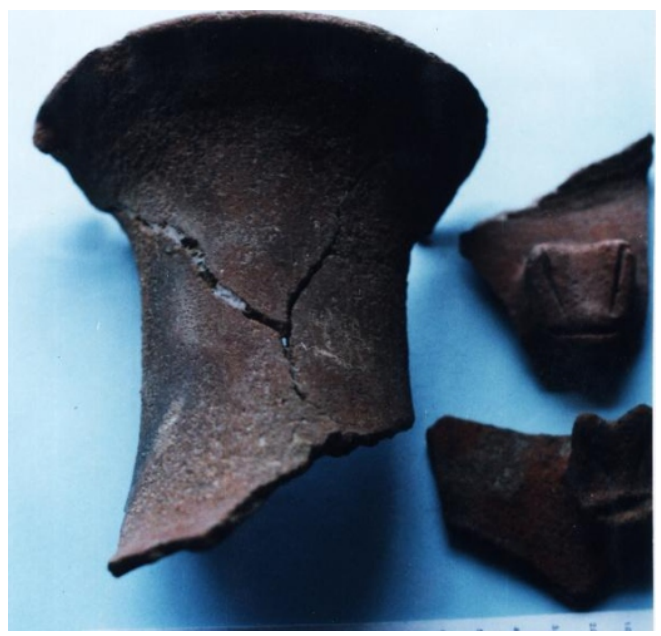

Figura 7. Cerámica utilitaria de "Los Hornos"

La cerámica utilitaria de "Los Hornos" es una cerámica muy rudimentaria (por su materia prima, acabado, tratamiento) pero sus formas y aplicaciones escultóricas son sin duda inca, es el Alfar 2.

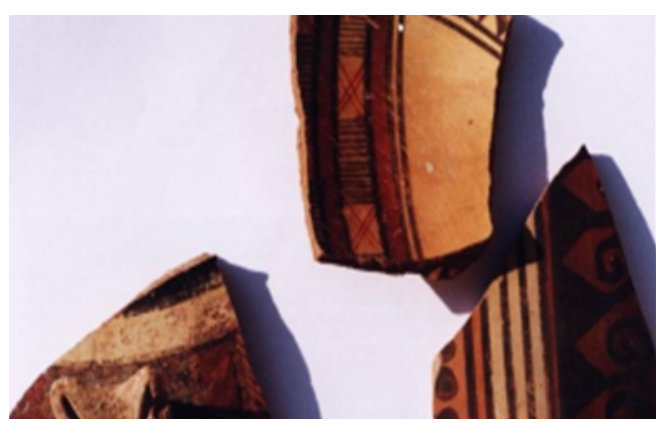

Figura 8. Cerámica de Los Hornos, "Imitación Cuzco".

\section{DISCUSIÓN}

En los valles del sur y su litoral, la presencia inca se manifiesta; y la introducción de la "mita" debió dinamizar la economía local. Sin embargo, el proceso de "incanización" se limitó debido a que las poblaciones locales mantuvieron sus identidades, de esa manera se sustentaba la complementariedad económica y gracias a transacciones económicas se lograba mayor productividad (Vela, 1999 ; Vela, 2005; Trimborn, 1975 ; Gordillo, 1996).

El sistema de enclaves que adoptó el estado inca no supone necesariamente una continuidad territorial, el objetivo no era conquistar territorios ni poblaciones, sino influir y hegemonizar en el modo de producción existente y hacerlo girar para sus intereses. Y, en función de su desarrollo agrícola, se instalaron en tierras aptas para el maíz, papa, yuca, legumbres, coca, ají; siendo los recursos del mar un atractivo especial, por el aporte a la alimentación que brindan las especies marinas.

Las excretas de aves marinas, el guano de isla, fue aprovechado como fertilizante en la agricultura, así se diseñaron estratégicas para incluir estos espacios a su orden económico. Desde tiempos tempranos se establecieron en la costa dos actividades diferentes: la pesca y la agricultura. Se formaron grupos separados con jefes propios y se estableció entre ellos un intercambio de productos. Sin embargo, los pescadores, limitados a sus playas y caletas, permanecieron subordinados a los señores de las macro etnias de agricultores yungas (Rostowrowski,1988).

La complementariedad no solo es económica, también tiene una dimensión étnica, política e ideológica. La complementariedad modeló relaciones recíprocas de intercambio, generando sistemas de movilidad y tránsito, estableciéndose relaciones entre los pueblos y permitiendo una economía basada en las potencialidades de cada ecología.

En ese sentido, los valles de la costa están dispuestos desde sus nacientes en la cordillera a casi 4,000 msnm hasta su desembocadura en el 
mar. De esa forma, los grupos étnicos se disponen en espacios ideales para el pastoreo de camélidos en la Puna, en valles interandinos donde es posible una óptima producción agraria con productos como la papa, la quinua, la coca, las maderas; valles medios donde el maíz es su principal exponente, además del ají, los frijoles, el algodón, los árboles frutales. Así se instalaron con patrones de asentamiento diseñados en función de su desarrollo económico.

El proceso de complementariedad en los andes fue estudiado desde la óptica de la etnohistoria (Murra, 1975; Rostowrowski, 1988) y por la arqueología con los trabajos de Mujica et al (1983), Stanish (1990) Muñoz et al, (1987) y Scchiappacasse et al (1989).

Cuando los incas se instalan en el valle de Sama se encontraron con una población diseminada en todo el valle, además del litoral. En las estribaciones de Sama se tiene varios sitios con evidencia inca (Gordillo, 1996), en la zona de valle serrano, en Sitajara, Yabroco. En la parte media del valle se tiene varios incas de "Sama la Antigua", "Yalata", "Pampa Julia" (Trimborn, 1975), estos sitios están relacionados a campos agrícolas, este valle es valioso por su producción de maíz, algodón, zapallos, calabazas, ají; además de árboles como el chañar y el molle que brindan maderas.

El litoral de Tacna posee una serie de sitios como "El Calvario", "Boca del Río", "Tomollo", "Los Hornos", "Quebrada de Burros", "La Lobera", "Punta Meca", los cuales presentan materiales de los estilos San Miguel, Pocoma, Gentilar y Maytas, es decir, fueron poblaciones con un complejo componente étnico, varias identidades inter actuantes.

"Los Hornos" debió ser un punto estratégico en la economía inca en la zona, además de su ubicación geográfica, pues siempre los sitios incas están instalados en lugares estratégicos, por lo que "Los Hornos" fue parte de una planificación económica y socio-política, de tal forma que las poblaciones incas del valle de Sama aprovechaban la agricultura. Además, "Los Hornos" estaba relacionado con la actividad pesquera, planificando el control de pisos ecológicos en el valle de Sama.

La presencia inca en el litoral de Tacna manifiesta que las poblaciones locales permanecieron con sus identidades, la población inca no estuvo interesada en lograr una total asimilación al incario, pues así realizaban transacciones comerciales y consolidaron sus influencias políticas que lograrían una actividad económica más dinámica.

Realizadas las exploraciones en el sitio, observamos que esto no es adyacente a zonas productivas (campos agrícolas, ganadería, caleta de pescadores, etc.) Sin embargo, se encuentra en una ubicación geográfica estratégica, pues puede controlar el litoral que estaba poblado por grupos de pescadores locales. Además, que desde "Los Hornos", la comunicación con el valle de Sama es más rápida, hay una serie de senderos que comunican el sitio con el valle y el litoral; esto, los materiales arqueológicos y la deposición en los basurales sugieren que "Los Hornos" fue un lugar donde se realizaban transacciones económicas y tenían un carácter de enclave que tenía un objeto económico.

"Los Hornos" presenta un patrón de asentamiento que difiere a los sitios que se encontraban en la costa de Tacna y que corresponden a los llamados grupos locales del periodo Intermedio Tardío, pues estos sitios están ubicados en la línea de playa, en las desembocaduras de ríos, mientras que "Los Hornos" se asentó en la cima de un cerro, este define otra estrategia y otro sistema de relaciones culturales. La recurrencia poco significativa de materiales de los Grupos Locales establece la identidad y la filiación del sitio "Los Hornos", y define la asociación cultural.

La presencia significativa de coprolitos de camélido en los basurales nos señala que existieron muchos animales vivos, los que debieron servir para el transporte de productos del mar hacia el valle y viceversa.

"Los Hornos" es un sitio con indicadores de la presencia inca en Tacna, sin embargo, esta presencia cultural fue ligera, es decir, no se 
produce un total proceso de "incanización", pues los grupos locales coexisten y desarrollan relaciones de complementariedad con la población inca.

La información arqueológica referida a la prehistoria tardía en el valle de Sama señala a la presencia inca como uno de sus componentes. Este periodo desarrolló un poblamiento multiétnico entre las poblaciones locales, la presencia inca y la presencia altiplánica, en este estudio el método analítico adecuado a la ciencia arqueológica nos permite reflexionar acerca de la presencia inca y las interacciones con la sociedad local, estableciendo coeficientes de regresión, efectos principales, interacciones, así como estimaciones de respuesta en superficie. Dicho análisis concuerda y refuerza estudios anteriores y plantean nuevas hipótesis de trabajo, pero en general sugieren que la presencia inca si bien es cierto significó un limitado proceso de "incanización", se observa que la curva de optimización de la presencia inca tiene una tendencia a decaer, mientras que la sociedad local manifiesta una tendencia a permanecer. El análisis descriptivo contribuye con cifras porcentuales que concuerdan con el desarrollo del método analítico. La presencia inca en el litoral de Tacna manifiesta que las poblaciones locales permanecieron con sus identidades, pues la población inca no estuvo interesada en lograr una total asimilación al incario, pues así realizaban transacciones comerciales y consolidar sus influencias políticas que a la postre logrará una actividad económica más dinámica, además de un uso sostenido de los recursos del valle de Sama.

\section{CONCLUSIONES}

Los Hornos fue un sitio que controlaba y mantenía relaciones socioeconómicas con las poblaciones locales e incaica, en el litoral, su planificación espacial responde a una estrategia de expansión y de interacción étnica.

La presencia inca en la costa de Tacna se manifiesta en el sitio Los hornos, con una cerámica inca de fabricación local con atributos de la cerámica "inca imperial" o "Cuzco policromo", que denominamos "imitación Cuzco".
Las sociedades de la prehistoria tardía en el valle de Sama lograron un desarrollo sostenido en base a la complementariedad y el medio ambiente, las poblaciones locales no pudieron ser "incanizadas" por lo sólido de su etnicidad y relaciones interétnicas en las sociedades locales. Este sistema desarrolló relaciones armónicas, flexibles y muy dinámicas entre la población local y la población inca.

En la cuenca del Sama, la población inca y la población local logran sostenibilidad, al desarrollar una economía muy compleja, aprovechando los recursos del mar en la pesca y recolección, así como los recursos del valle para la agricultura, pastoreo, recolección, además de actividades artesanales.

La sostenibilidad entre las sociedades en la prehistoria tardía en el valle de Sama se sustenta en las interacciones entre la complementariedad y las relaciones ambientales del valle de Sama. Este valle presenta una variedad de paisajes, pisos ecológicos, microclimas, zonas productivas, zonas potenciales, así como una biodiversidad que permitió optimizar la producción y lograr un desarrollo sostenible.

En las tablas presentadas, podemos observar la diversidad de materiales arqueológicos en contexto del sitio "Los Hornos". Ubicado en la costa, la mayoría de materiales son de origen de valle o Yunga, como de la zona quechua, además de un significativo porcentaje de materiales marinos. Este es un indicador de los procesos de complementariedad e intercambio entre las sociedades de la prehistoria tardía.

Se observa que en los materiales arqueológicos hay una tendencia a los de origen vegetal como animal y en menos porcentaje los de origen mineral. En concordancia con la actividad económica de su población, eran grupos de agricultores, pescadores, ganaderos y artesanos que aprovechaban los recursos del mar, del valle, las lomas, la puna y los recursos minerales que brindaba el medio ambiente.

Los exámenes realizados nos demuestran que no existió fusión entre las sociedades 
intervinientes; que la sociedad étnica tiene una curva de optimización muy débil en comparación con la sociedad local, el análisis de Pareto indica que prevalece la sociedad local.

\section{REFERENCIAS}

Barriga, V. (1946). Memorias para la Historia de Arequipa. Lima: La Colmena.

Cúneo Vidal, R. (1977). Historia de los Cacicazgos Hereditarios del sur del Perú en Obras Completas de Ignacio Pastor. Lima: Gráfica Morsom

Galdós G. (1985). Interrelaciones estructurales en la costa sur peruana. Diálogo Andino $\mathrm{N}^{\mathrm{o}} 3$ Universidad de Tarapacá. Arica.

Gordillo, J. (1996). Desarrollo regional tardío y ocupación Inka en la pre-cordillera de Tacna. Ciencia y Desarrollo (3). Universidad Nacional Jorge Basadre Grohmann de Tacna.

Lumbreras, L. (1974). Los reynos postTiwanaku en el área altiplánica. Revista Museo Nacional, 40.

Mujica, E. Rivera, M. y Lynch, T. (1983). Proyecto de estudio sobre la complementariedad en los valles occidentales del área centro-sur andina. Chungará(11). Universidad de Tarapaca Arica, Chile.

Murra, J. (1975). Formaciones económicas y políticas en el mundo andino. Lima: IEP.

Muñoz, I. (1981). La aldea del cerro Sombrero en el periodo del Desarrollo Regional de Arica. En Chungará $\quad \mathrm{N}^{\circ} 7$, Universidad Nacional del Norte. Arica.

Muñoz, I. Chacama, J., Espinoza, G. y Briones, L. (1987). La ocupación prehispánica tardía en y su vinculación a la organización social y económica inca. Chungará (18).

Universidad de Tarapacá. Arica, Chile.

Rice, D., Conrad, G. y Watanabe, L. (1990). Proyecto Osmore: Un estudio del Programa Contisuyo sobre la complementarieadad en la prehistoria tardía en la cuenca del Osmore, Moquegua, Perú. Lima: Escuela Nueva.

Rostowrowski, M. (1988). Recursos naturales renovables y pesca, siglos XVI y XVII. Lima: Instituto de Estudios Peruanos.

Schiappacasse, V. y Niemeyer, H. (1989). Avances y sugerencias para el conocimiento de la prehistoria tardía de la desembocadura del valle de Camarones (Región de Tarapacá). Chungará (22). Arica.

Stanish, C. (1990). Complementariedad zonal en Moquegua: una aproximación desde el valle de Moquegua. Gaceta arqueológica andina (18/19). Lima: INDEA.

Trimborn, H. (1975). Investigaciones arqueológicas en los valles del Caplina y Sama. Navarra: Verbo Divino Estrella.

Vela, C. (1999) Estudio de la presencia e impacto inka en el sitio Cerro Los Hornos, Tacna: a través de su Cerámica. Tesis, Universidad Católica Santa María de Arequipa.

Vela, C. (2005) Complementariedad y Medio Ambiente como Base del Desarrollo Sostenible en las sociedades de la prehistoria tardía en el valle de Sama: el caso del sitio arqueológico "Cerro Los Hornos" (tesis de maestría). Universidad Jorge Basadre Grohmann. Tacna. 